\title{
Anatomy for Clinical Terminology
}

\author{
Alan L Rector
}

Summary. Anatomical notions provide the foundations for much clinical terminology. However, clinicians' concerns are notoriously practical. The goal in clinical terminology is not to represent anatomy for its own sake, but rather to facilitate faithful communication by clinicians about what they have heard, seen thought and done in their care of patients. The focus of clinical anatomy is therefore the interaction between the locus of disease and partonomy - that diseases of parts are, with specific exceptions, diseases of the whole. Clinical anatomy must also accommodate the clinical/functional view as well as the structural view basic to classic anatomy. One means of doing so in current ontology formalisms is through a hierarchy of relations. There are a number of problems where there is no consensus on solutions. Of particular importance are the relations of tissues and substances to structures and representation of characteristics collective effects of entities such as cells, One approach to this issue is discussed here. Finally, there is currently no expressively adequate and computationally tractable means for expressing abnormal and variant anatomy. All resources are explicitly about "normative anatomy". In dealing with all of these problems it is essential to separate issues of "terminology" - i.e. the labeling of entities from their formal definitions and relations. Clinicians frequently use terms that anatomist now consider obsolete or deprecated. However, a distinction should be drawn between the mere renaming of an entity - however well motivated - and more fundamental revision as to its structure or function.

\subsection{Introduction}

Anatomical notions provide the foundations for much of clinical terminology. Anatomy is a key part of the fundamental vocabulary, and it is not possible to discuss disorders and treatment of the body without using anatomical notions.

However, clinicians' concerns are notoriously practical. They might recognise the same underlying foundations in anatomical structure embodied in the Foundational Model of Anatomy [6, 15]. They might assent to the formal descriptions of part-whole relations put forward by Smith et al. on behalf of the Open Biomedical Ontologies (OBO) consortium [19]. However, what they use day-to-day is a mixture of structure, function, and convenience. The OpenGALEN anatomy schemata were

Status:

- editing complete;

- CPR form received; 
created to address issues this clinical level of abstraction. They are well documented in several publications $[10,11,14]$ They have also been compared with the Foundational Model of Anatomy (FMA) [7, 8, 22]. We will not describe them in detail here. Rather this chapter will use them to illustrate key issues that must be addressed by any formal representation of anatomy that seeks to be used in clinical applications.

The goal in clinical terminology is not to represent anatomy for its own sake, but rather to facilitate faithful communication by clinicians about what they have heard, seen, thought and done concerning patients and their conditions. Because clinicians are concerned primarily with disorders and dysfunctions, any representation of anatomy for clinical purposes must deal with function as well as structure and development. Because a significant part of clinical terminology deals with pathology, any terminology for clinical anatomy must deal with the arrangements of tissues as well as with organs and other structures.

Our goal is a logical foundation for clinical terminology which is sufficient as a reference point for clinical terminologies. With respect to anatomy, there are five key goals, each of which will be discussed in turn:

- "Locus" and Partonomy. The most important relationship between clinical medicine and anatomy is in specifying the locus for disorders and procedures. The fundamental internal relation in anatomy is partonomy. The interaction between partonomy and locus is therefore critical to using any formal ontology of anatomy in clinical medicine. This includes issues of understanding when partonomy does not appear to be treated as transitive.

- Reconciliation of Clinical/Functional and Structural Views. How to reconcile the classical structural view of anatomy and the more functional and pragmatic view used for reasoning in clinical medicine. The challenge in a more generic anatomy is to accommodate the clinical view without distorting the "pure" view of the structural anatomist and developmental biologist.

- Tissues, substances and structures. Clinical descriptions of organs of the tissues that constitute them are performed by different specialists in attending to different features using different vocabulary. Developmental anatomists tend to focus on tissues whereas clinicians and anatomists concerned with adult organisms tend to focus on structure.

- Abnormal anatomy and congenital anomalies. Classical anatomy is "normative". It deals with what is typically true. Clinical practice must deal with the myriad variations and distortions that occur in practice. Representation of normal and abnormal anatomy and the relationships between various abnormalities is a major unmet challenge.

- Synonyms and variant terminology. Issues of terminology and synonyms is as prevalent in anatomy as in any other biological field. Differences occur over time and between different academic communities and between different geographical locales. In particular, clinical usage is often different from formal anatomical 
terminology. Hence any anatomical terminology, needs to be able to cater for alternative terminology.

A set of ontologies formulated in OWL demonstrating these principles can be found at http://www.co-ode.org/ontologies under "Sample Top Bio".

\subsection{Locus and Partonomy - The Basis of Clinical Reasoning about Anatomy}

The most important function for anatomy in clinical terminology is as the locus for disorders. Many diseases clearly manifest at an anatomical location or in an anatomical structure - e.g. "fracture of femur", "tumour of lung" - or in anatomically related notions - e.g. "bronchogenic carcinoma". Other notions notions are so closely tied to anatomy that they are usually described as localised to some particular organ even though the manifestations may occur remotely or affect the body globally $-e . g$. "hypothyroidism" or "cerebellar ataxia". Signs and symptoms are almost by definition related at least loosely to anatomical notions, even when they are vague terms such as in "indigestion". The pattern is so ubiquitous that many clinical nomenclatures, including some versions of the original SNOMED pathology codes, require an anatomical locus ${ }^{1}$; where there is no specific locus, some construct such as "body as a whole" is often used.

However, since the relation between disorders and anatomy is not strictly speaking one of physical location, it is preferable to use a label such as "locus" for the relation rather than any term which implies physical location. Nor is it useful to define the notion of "locus" to tightly. For example the exact relation between disorder and anatomy is different in "pneumonia", "pleuridinia", "intestinal obstruction", and "cholelithiasis"; however, the differences are not consistently reflected in language, and each condition would normally be classified under disorders of the anatomical structures involved. In effect the "locus" relationship is defined pragmatically as the disjunction of all of these relations and others, which are not distinguished in common clinical parlance. ${ }^{2}$

The fundamental relation of anatomy is the part-whole relation - the foot is part of the lower extremity; the ventricle is part of the heart, etc. The key pattern for clinical anatomy relates "locus" and "partonomy".

The fundamental pattern is that, with few exceptions, disorders of parts are disorders of the whole. For example, a fracture of the neck of the femur is considered a kind of fracture of the femur; stenosis of the aortic valve is considered as a heart disease; cataracts, which occur in the lens, are considered a disorder of the eye, etc.

\footnotetext{
${ }^{1}$ now the "site" qualifier in SNOMED-CT

${ }^{2}$ OpenGALEN used the label "location" and came to regret the confusion caused.
} 
The same is typically true of procedures. Fixation of the neck of the femur is an operation on the femur; repair of the aortic valve is a heart operation; removal of a cataract is an operation on the eye, etc. ${ }^{3}$

Put another way, when we use the phrase "Disorder of X", what we usually intend is "disorder that has locus X and/or any of its parts". Likewise, when we speak of "Procedure on X", we usually mean a "procedure that has locus X and/or or any of its parts".

There are two principled exceptions:

- Effects on entites "as a whole" - e.g. amputations, where we would not consider the amputation of a finger to be an amputation of the hand although we would consider an injury to the finger to be an injury to the hand. There is a limited but important set of constructs such as "Amputation of X" that should not be interpreted as applying to "X and/or its parts".

- Collective effects - e.g. the collective failure of the pancreatic islet cells in type one diabetes. Collectives are a more complex case that is discussed under 3.4.2 below.

The standard pattern of a "disorder a structure and/or any of its parts" gives rise to standard transformation whereby separate hierarchies of kinds and parts give rise to a combined hierarchy of disorders as shown in Figure 3.1. Because this transformation is so common, it is also common to confuse the hierarchies of parts and wholes with the kind-of or subsumption hierarchy. The two are distinct, but related through the common patterns shown in Figure 3.1.

These notions are easily represented in any logic based formalism supporting disjunction (the logical operator "or"), including the new web ontology language, OWL. ${ }^{4}$ Alternatively, even without disjunction, any system supporting inheritance hierarchies can achieve a similar effect by rewriting each structure as a "SEP triple" $[4,16,17]$ consisting of: i) a "Structure" node for what we have called the reflexive parts of the entity, ii) an "Entire" node, for the entity itself, and iii) a "Part" node for the proper parts as shown in Figure 3.2. This transformation is possible even in relatively weak formalisms and, correspondingly, leads to highly computationally efficient representations. (Normally, the transformation is hidden so that users need not be aware of the extra entities.) The SEP triple transformation makes it easy to see the interaction between the locus relation and the kind of relation as shown in Figure 3.3. If we interpret "Disorder of Heart" as "Disorder of Heart of its parts"; interpret "Disorder of Ventricle" as "Disorder of Ventricle or its parts", etc., then it follows

\footnotetext{
${ }^{3}$ This is not true just of medicine, but of our conceptualisation of the world in general. For example, a fault in a fuse is considered to be a fault of the electrical system, which is in turn considered to be a fault of the car.

${ }^{4}$ For a discussion of how to formulate such expressions in OWL see http://www.w3.org/2001/sw/BestPractices/OEP/SimplePartWhole/index.html.
} 


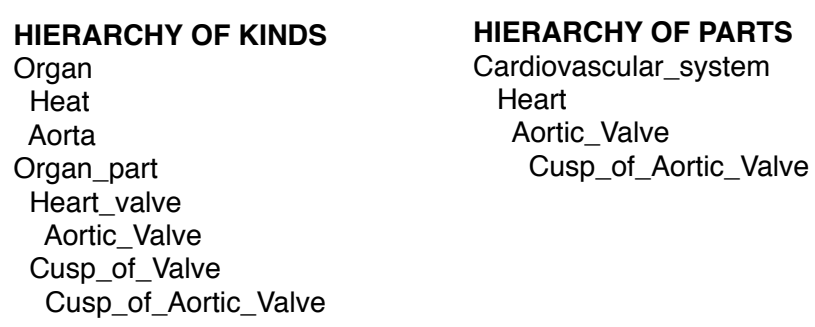

RESULTANT HIERARCHY OF KINDS OF DISORDERS

Cardiovascular_disease

Heart_disease

Valvular_heart_disease

Aortic valve disease

Disorder_of_cusp_of_Aortic_valve

Fig. 3.1. Interaction of Hierarchies of parts and kinds

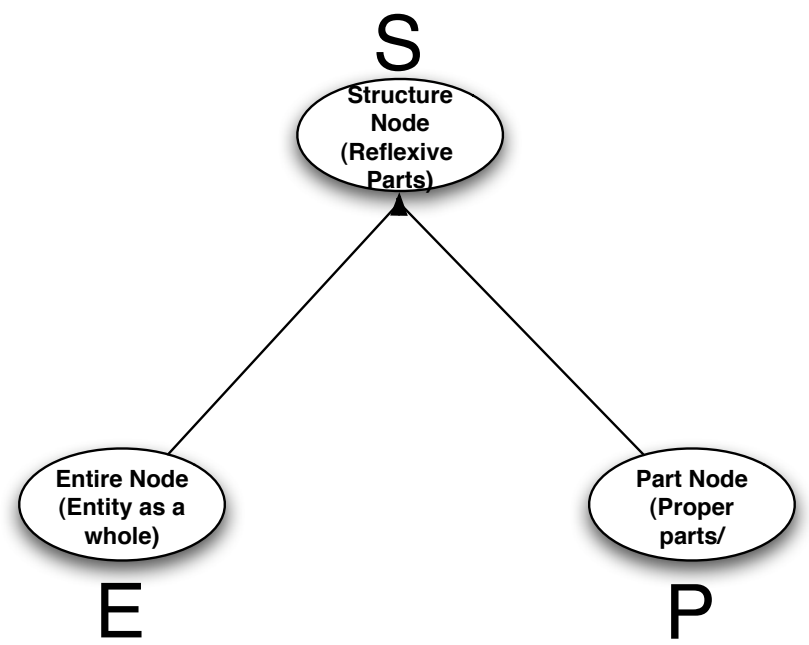

Fig. 3.2. SEP Triple

immediately that a disorder of a part of the ventricle, say the "Aortic Valve" can be inferred to be a disorder of the Ventricle and therefore of the Heart. 


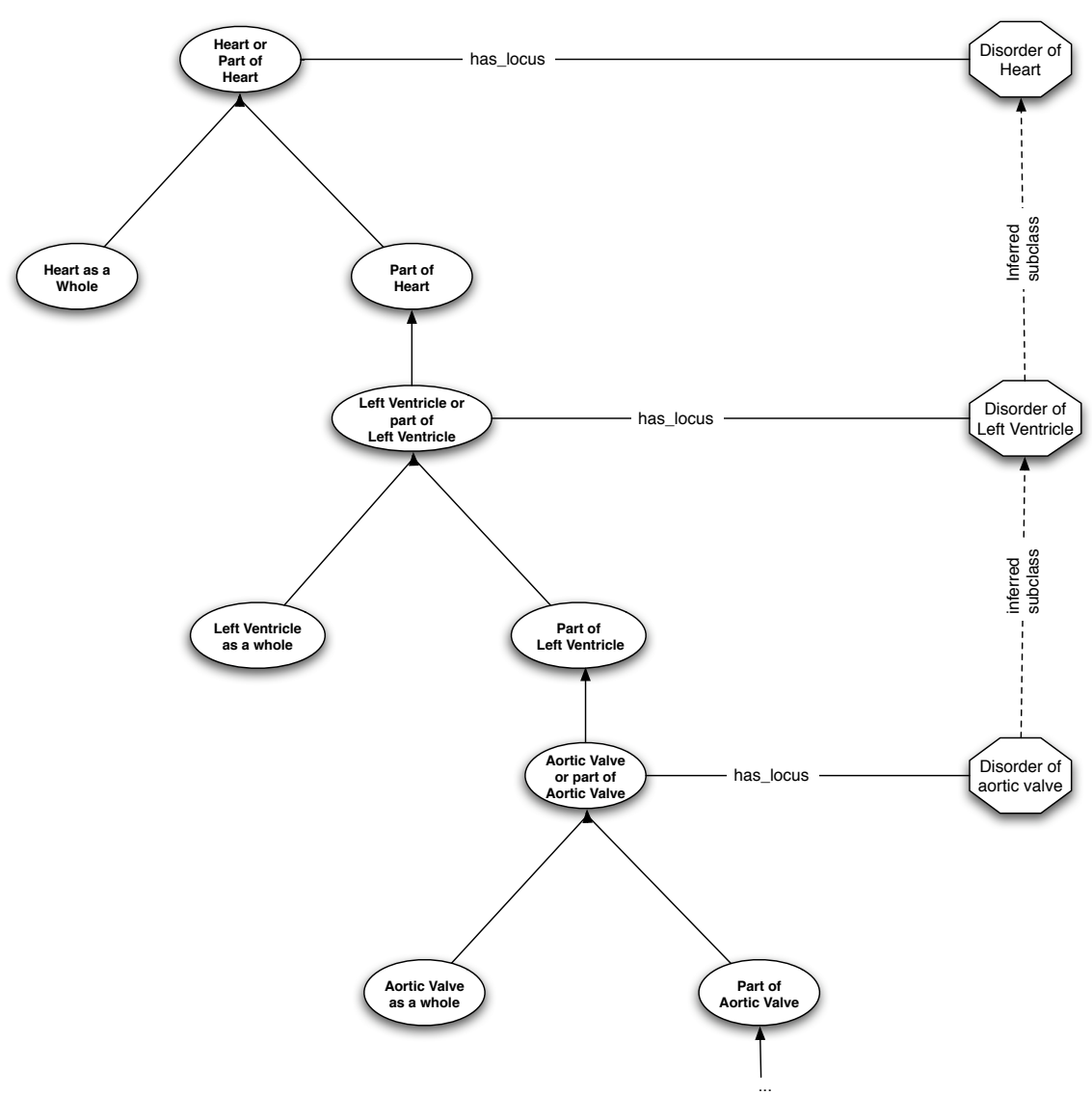

Fig. 3.3. Example hierarchy of SEP triples and interaction with locus and inferred hierarchy of disorders

\subsection{Accommodating Clinical/Functional and Structural Views - using a Hierarchy of Relations.}

Classical structural anatomy is defined in terms of physical structure and developmental morphology. However, reasoning with these structures in clinical medicine normally involves their functions. We want to preserve the notion that the disease hierarchy results from an interaction of locus and partonomy even when the part-whole relation does not conform, strictly, to the structural notions of classical anatomy.

The paradigmatic case is the pericardium. No clinician would dispute that, in terms of embryological development, the pericardium is a separate organ from the heart. However, few clinicians would expect to find pericarditis classified elsewhere than under cardiac disorders, and most clinicians would expect a comprehensive dis- 


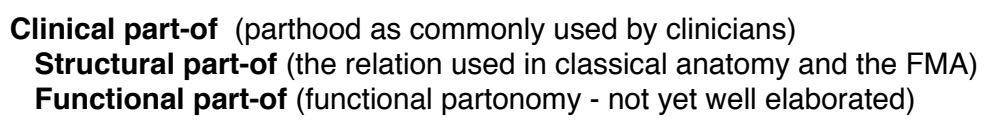

Fig. 3.4. A fragment of the hierarchy of partonomy relations showing clinical partonomy subsuming functional partonomy

cussion of malfunctions of the heart to include pericarditis ${ }^{5}$ and cardiac tamponade ${ }^{6}$.

For these clinical purposes, the pericardium behaves functionally as part of the heart. The challenge in a more generic anatomy is to accommodate the clinical view including function without distorting the "pure" view of the structural anatomist.

We can achieve this if we regard the notion of "clinical parthood" as a more general relation of classical structural parthood. Most logic based formalisms support a hierarchy of relations and subrelations. ${ }^{7}$ In any such formalism we can define a hierarchy of relations as shown in Figure 3.4 [13]. We wish to say that anything which is structurally part of a whole is also clinically part of the whole, but not vice versa e.g. that the pericardium is clinically part of the heart but not structurally part of the heart. On the other hand, we want to say that the ventricle is clinically part of the heart because it is structurally part of the heart. This is precisely the meaning of stating that structural parthood is a sub-relation of clinical parthoood. In general, to say that one relation is a sub-relation of another, is to say that any two entities related by the sub-relation are also related by the super-relation - in the example given, that any two entities related by structural parthood are also related by clinical parthood. More succinctly, to say that structural parthood is a sub-relation of clinical parthood is to say that structural parts are clinical parts.

This allows us two "views" of parthood, the first using the structual parthood relation is confined to just those things that would be represented by anatomist; the second allows a broader definition to take in those things that would be included in clinical expressions typically expressed, at least informally, as parthood.

In the above, and in our practical developments, we have left "functional parthood" as a placeholder because we do not wish to prejudge a complex discussion which should probably be left for a reference model of biological functions and processes. In particular, whether or not structural parthood always implies functional parthood is not a debate we wish to enter into here.

\footnotetext{
${ }^{5}$ inflammation of the pericardium

${ }^{6}$ restriction of cardiac function because of fluid in the pericardial sac

7 "properties" and "subproperties" in OWL or "slots" and "subslots" in Protege
} 


\subsection{Tissues, Substances and Structures}

\subsubsection{Constituents: Tissues and Substances}

While some clinicians are concerned primarily with gross anatomical structures, pathologists and clinical histologists are concerned primarily with tissues and micro structures. Much of clinical medicine, particularly with respect to cancer and surgery, revolves around the description of tissues. However, it is notable that there is a recognised line between gross and micro anatomy, both in delineation of clinical specialities and in medical education. In addition there are a variety of substances blood, urine, sweat, etc. - that are also described by related specialists in related terms.

What should be the status of tissues and substances in a terminology of clinical anatomy? Clearly the information conveyed about them is different from that conveyed about gross structures. We say things like "Bone tissue is made up of matrix with osteocytes scattered sparsely throughout" which would make no sense of individual structures.

This issue is paralleled by a continuing debate amongst ontologists concerning how constituents should be represented. The argument is typically formulated concerning a "statue made of clay". The cognitivist or multiplicative approach taken by Guarino and Welty in DOLCE and OntoClean $[1,3,20]$ maintains that the clay and the statue should be represented as two different entities, and that the clay "constitutes" the statue. The argument is that if the statue is damaged and looses some clay and then repaired with different clay, it is still the same statue but a different mass of clay. Smith in BFO maintains that, since there can only be one physical object occupying the same space and time, the clay and the statue must be the same entity $[2,18]$. Smith resolves the problem of changing composition by indexing parthood by time and does not give identity the same central role in BFO that it has in DOLCE and OntoClean.

Translated to anatomy, the question is whether there is a distinction to be made between a lobe of the liver and the liver tissues that make up that lobe? Or more practically, whether there a distinction to be made between a "piece of liver parenchyma" and "liver parenchymal tissue".

The philosophical dispute cannot be resolved here. What is clear from a clinical point of view, is that different sorts of clinicians have different things to say to about tissues and structures. There is different information to be represented concerning structures and concerning tissues and substances. Furthermore, a pathologist examining a slide will describe it as "liver parenchymal tissue" or perhaps tissue even if it is for some reason "ectopic" - i.e. not found in the liver. In fact the whole notion of "ectopic tissue" makes no sense unless we can speak separately of the tissue and the structure that it would normally constitute. Hence, for purposes of a clinical representation for information systems, it is simpler to treat tissues and the structures they 
constitute separately, as in DOLCE.

We then label the relation between the structure and what makes it up as the "constitutes" relation, whose inverse we shall label "constituent" and which corresponds, roughly, to the use of "consituent part" in the FMA. Tissues and other constituents are analogous to mass nouns whereas structures are analogous to count nouns - we can say that we have one liver and two kidneys but only that the liver consists of roughly two kilograms of some mixture of tissues. Whereas structures have shape and spatial relationships, mass constituents have composition and arrangements. We can say that the liver is below the diaphragm but that the liver cells are arranged in roughly hexagonal patterns around bile canniculi. Or we can say that the proportion of collagen in liver tissue is of a given value or specify the percentage by weight of sodium ions in serum.

For completeness it is useful also to distinguish a "portion-of" relation between substances and mixtures of substances. We can then say that "substances" form portions of "mixtures" which constitute "structures".

\subsubsection{Collectives}

The issue of patterns brings us to the issue of collectives. A pathologist might say, for example, that this pattern was either intact or disrupted. In doing so he or she is referring not to the individual cells, or even to one example of the pattern, but to the overall collective appearance.

The individual cells in tissue, like the individual molecules in water and other substances, are of little concern. Loosing or gaining a discrete structure such as a finger matters clinically; losing or gaining a liver cell or a molecule of water in the interstitial fluid does not.

We are concerned with the collective effects and patterns of cells and molecules, rather than their individual effects. Hence we need to represent the collectives explicitly in the ontology. Typically we use the relation "is grain of - by analogy to grains of sand on a beach - to link the individual grains of a collective to the collective as a whole. There is a recurring pattern. Typically, discrete grains at one level form collectives that act as mass entities at the next, e.g. cells form (portions of) tissue, collectives of molecules form substances, etc. [9, 21].

Putting these two patterns together we have the larger pattern that collectives of grains form mass entities which constitute discrete structures. There are different things to be said at each level, for example of the individual molecules of collagen, of their arrangement connective tissue, and of the ligament constituted by the connective tissue. Frequently different information sources and experts are involved at each level. Maintaining each level separately allows the information system to be factored 


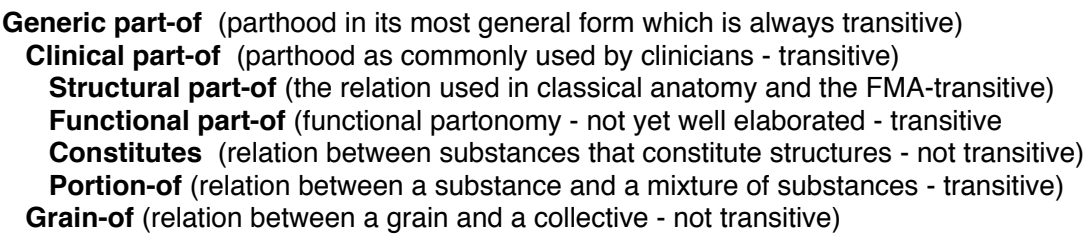

Fig. 3.5. A fragment of the hierarchy of partonomy relations showing clinical partonomy subsuming functional partonomy

so that each group can deal with it separately and relatively independently.

Note that grains are not sets. Sets are defined by their members. However, the members of collectives change constantly. For example, blood cells are constantly created and destroyed even when all measures of the collective "full blood count" remain constant. Cells are constantly lost from the skin and muscosa, and indeed most tissues without are considering it an injury. Indeed the failure of the normal turn-over represents an abnormality. The same applies to grains of sand on a beach or flocks of birds. The loss or gain of a few members does not affect the identity of the whole until that loss or gain affects the collective behaviour or function or appearance.

Note also that the notion of collective also provides a means of setting limits on the transitivity of locus across partonomy. A disorder of "a liver cell" is not normally treated as a disorder of the liver whereas a disorder of "liver cells" - i.e. a collective of liver cells - is considered as a disorder of the liver. The issue of when partonomy is, or is not, transitive is one which has long been debated by mereologists [5]. Many of the cases of interest can be explained if we simply state that, for most purposes, the transitivity of parthood stops at collectives.

Hence in 3.5, the "grain of" relation is not a subrelation of the "clinical partonomy" relation because being a grain of a collective that constitutes an entity does not imply being a clinical part of that entity. However, we provide a still higher level notion of partonomy - corresponding to the union of classical mereological partonomy, functional partonomy, and clinical partonomy - which does include the "grain of" relation. (A more detailed example can be found in [12].)

\subsubsection{Summary of Views on Structure, Function, Constituents and Grains: Extended Relations Hierarchy}

The issues of the differing views of clinical and structural anatomy, the different modes of constituents and structural parts, and the need to query the anatomy sometimes in a sense in which partonomy is considered always transitive and sometimes in a sense at which transitivity stops at the boundaries of grains and collectives can 
all be captured using a hierarchy of relations as shown in Figure 3.5. Since anything true of any of the subrelations is also true of the super-relations, a query using the most generic part-of will include all structural, functional parts, constituents, portions, and grains transitively. Clinical partonomy includes all relations but grains; structural partonomy just classical structural parts. If there is a need to include a form of parthood that includes both structural parthood, constituents and proportions, this can be accommodated by further elaborating the hierarchy of relations. Although not all disputes can be resolved in this way, developing a hierarchy of relations is a powerful methodology for accommodating multiple views.

\subsection{Abnormal Anatomy and Congenital Abnormalities}

Most anatomy references deal primarily with normal anatomy. The FMA is explicit in being a reference of "normative anatomy." However, much of clinical medicine concerns abnormal anatomy. Dealing with abnormal anatomy raises a series of issues:

- The notion of "normal" and "abnormal" are difficult to define. In particular do they refer to whether or not a structure is "normative" or "typical", whether or not it performs its usual function, or whether or not it is clinically significant?

- The notion of "normative" is probably distinct from "normal" in the clinical sense. Most references of anatomy, notably the FMA describe themselves explicitly as references on "normative" anatomy.

- The notion of "normality" is applied both to structures as a whole and to features of those structures. If applied to structures as a whole, it may be questionable as to whether any real structure even corresponds completely to the "normative" ideal.

- Notions of "normative" are essentially meta-level notions which relate an individual to the type or class of such individuals. For example, whereas we can say that a hand has five fingers without reference to other hands, we can only say that it is "normal" in relation to other hands.

- Interrelations amongst abnormalities are common. Multiple congenital anomalies often spring from a single cause.

The simplest notion of normality, and perhaps the most useful from a clinical point of view, is pragmatic. A structure is "abnormal" if it is somehow clinically noteworthy and not otherwise. To a first approximation, it is abnormal if a clinician would consider it worth noting in a medical record. GALEN's distinguished two notions pragmatically, motivated by the need to make sense of the common clinical construct "abnormal but not pathological":

- Normal vs NonNormal - whether or not some feature or structure was worth noting in a clinical record.

- Pathological vs NonPathological - whether or not some feature or structure required medical management in some sense (including continued monitoring). 
This pragmatic formulation has two immediate advantages. Firstly, it supports the expected inferences for clinical practice and statistics. Secondly, it is strictly first order. $^{8}$ It refers to the individual structure itself without relying directly on any notion of the class. It is a statement that a particular structure is noteworthy or in need of attention, without commitment as to why. In general it also follows the pattern that if a part is non-normal or pathological, then the whole is likewise non-normal or pathological.

Other notions of normality are more problematic and intrinsically higher order. A closely related notion is "typical" which illustrates the practical consequences of such notions being higher order rather than first order, i.e. referring to the class itself rather than to all of the individuals in the class. Such meta-notions are not inherited in the usual way. Consider, for example, the case of mammalian red blood cells. "Typical" mammalian cells have nuclei; typical mammalian red blood cells do not; therefore they are not kinds of typical mammalian cells. However, abnormally red cells may have nuclei, so that certain abnormal mammalian red cells may be classified as kinds of typical mammalian cells in this respect.

As the example of mammalian red blood cells illustrates, the notion of "normative" is intimately tied up with issues of defaults and exceptions. It is slightly easier to manage if we attach the notion of normality not to the structure but to the feature in this example to "having a nucleus" rather than to the cell type. If we attach the notion of normality to the feature rather than the entity, then the standard frame style default and exception mechanism appears to work reasonably well, but to the best of our knowledge has not been carried through on any large scale for any anatomical reference resource.

In summary, at a pragmatic level, a notion of "abnormality" that amounts to flagging a specific entity as noteworthy is relatively simple and easy to implement. Deeper notions of normative anatomy and abnormal development present difficult conundrums and are beyond the bounds of current formalisms and the scope of this brief chapter.

\subsection{Synonyms and Variant Terminology}

As in any other terminology, a clear distinction is required between the linguistic "term" and the underlying "concept" or "entity" that it labels. The various clinical terminologies such as SNOMED and ICD each have idiosyncrasies of anatomical naming. There are marked differences between classic references such as Nomina Anatomica, the Foundational Model of Anatomy, and various model organism anatomical references. Furthermore, common clinical usage is often at variance with

\footnotetext{
${ }^{8}$ More accurately it is "epistemic", i.e. it refers to the clinician's understanding of the specific structure. However, most clinical usage is "epistemic" in this sense.
} 
any official anatomic nomenclature and usually lags behind official changes.

Because the use of anatomical terms in clinical terminologies brings together different disciplines, problems of linguistic labelling often give rise to disputes. In resolving such disputes it is essential to distinguish whether the issue is the label or the underlying anatomical concept. For example, it is clear that the reclassification of the Thymus as an organ of the immune system rather than an endocrine organ is substantive. It is less clear that the renaming of the "left anterior descending artery" as the "anterior intraventricular artery" has any such substantive import or merely represents an improvement in linguistic consistency. Indexing and labelling of clinical resources need to provide both common clinical usage and various official preferred usages.

\subsection{Summary}

Anatomy is central to clinical terminology, but the notions of anatomy are pragmatic and functional. The clinician's pragmatic view is often at variance with the strict "biological" view of the anatomist or developmental biologist. The key issue for clinical terminology is that, with certain well defined exceptions, disorders and procedures of parts are considered as kinds of the corresponding disorders or procedures of the whole.

It seems likely that many, if not most, of these differences between clinicians on the one side and anatomists and developmental biologists on the other can be overcome by treating clinicians' pragmatic notion of partonomy as an abstract superrelation and the more biologically motivated notions of the anatomist and developmental biologist as subrelations. However, this has not yet been demonstrated on a large scale.

It is essential in clinical anatomy to distinguish between discrete parts and pieces common in gross anatomy and the patterns and arrangements in micro-anatomy. This is is not a matter of size but of whether individual or collective effects are of concern. Cells usually functional collectively; hands, and hearts usually functional individually. This remains an area of controversy in which no complete solution is forthcoming.

As long as these issues remain, it is likely that the anatomy for clinical resources will remain distinct from major reference anatomies. How much this is a matter of principle, and how much a matter of inertia only the future will tell.

\section{References}

1. Aldo Gangemi, Nicola Guarino, Claudio Masolo, Alesandro Oltramari, and Luc Schneider. Sweetening ontologies with dolce. In A Gmez-Perez and V Richard Benjamins, edi- 
tors, European Knowledge Aquisition Workshop (EKAW-2002), pages 166-181, Siguenza, Spain, 2002. Spring Verlag.

2. Pierre Grenon, B Smith, and L Golberg. Biodynamic ontology: Applying bfo in the biomedical domain. In D M Pisanelli, editor, Ontologies in Medicine, pages 20-38. IOS Press, Amsterdam, 2004.

3. Nicola Guarino and Chis Welty. An overview of ontoclean. In S Staab and R Studer, editors, Handbook of Ontologies, pages 151-159. Springer Verlag, 2004.

4. Udo Hahn, Stefan Schulz, and Martin Romacker. Partonomic reasoning as taxonomic reasoning in medicine. In Proc. of the 16th National Conf. on Artificial Intelligence \& 11th Innovative Applications of Artificial Intelligence (AAAI-99/IAAI-99), pages 271276, Orlando FL, 1999. AAAI Press/MIT Press.

5. Ingvar Johansson. On the transitivity of parthood relations. In H. Hochberg and K. Mulligan, editors, Relations and Predicates, pages 161-181. Ontos Verlag, Frankfurt, 2004.

6. J L V Mejino and C Rosse. Conceptualization of anatomical spatial entities in the digital anatomist foundational model. Journal of the American Medical Informatics Association, (1999 Annual Symposium Special Issue):112-116, 1999.

7. Peter Mork and Philip Bernstein. Adapting a generic match algorithm to align ontologies of human anatomy. In 20th International Conference on Data Engineering, Boston, 2004. IEEE.

8. Peter Mork, Rachel Pottinger, and Philip Bernstein. Challenges in precisely aligning models of human anatomy. In Marius Fieschi, Enrioco Coiera, and Yu-Chuan Jack Li, editors, Proceedings of Medinfo 2004, pages 401-405, San Francisco, CA, 2004. IMIA.

9. James J Odell. Six different kinds of composition. Journal of Object Oriented Programming, 5(8):10-15, 1994. A clear presentation, apparently taken from Winston et al 87. Distinguishes: component-integral object, material-object (made-of), portion-object (slice of bread, loaf), place-area (city-state), member-bunch, member-partnership (as a kind of member-bunch). These he distinguishes from non-partitive relations: topological inclusion (seems confussed to me with place-area), classification inclusion (i.e. kind-of), attribution, attachment, ownership. Then gives smashing examples of transitivity and shows how the transitivity of each type is distinct.

10. AL Rector, A Gangemi, E Galeazzi, AJ Glowinski, and A Rossi-Mori. The galen core model schemata for anatomy: Towards a re-usable application-independent model of medical concepts. In P Barahona, M Veloso, and J Bryant, editors, Twelfth International Congress of the European Federation for Medical Informatics, MIE-94, pages 229-233, Lisbon, Portugal, 1994.

11. A.L. Rector and J.E Rogers. Ontological and practical issues in using a description logic to represent medical concept systems: Experience from galen. In P Barahona, F Bry, E Franconi, N Henze, and U Sattler, editors, Reasoning Web, LNCS 4162, pages 197231. Springer-Verlag, Heidelberg, 2006.

12. Alan Rector, Jeremy Rogers, and Thomas Bittner. Granularity, scale \& collectivity: When size does and does not matter. Journal of Biomedical Informatics, 39(3):333-349, 2006. httxp://dx.doi.org/10.1016/j.jbi.2005.08.010.

13. Alan L Rector. Defaults, context and knowledge: Alternatives for owl-indexed knowledge bases. In Russ B Altman, A Keith Dunker, Lawrence Hunter, Tiffany A Jung, and Teri E Klein, editors, Pacific Symposium on Biocomputing (PSB-2004), pages 226-238, Kona, Hawaii, 2004. World Scientific.

14. JE Rogers and AL Rector. Galen's model of parts and wholes: Experience and comparisons. Journal of the American Medical Informatics Association, ((Fall symposium special issue)):819-823, 2000. 
15. C Rosse, I G Shapiro, and J F Brinkley. The digital anatomist foundational model: Principles for defining and structuring its concept domain. Journal of the American Medical Informatics Association, (1998 Fall Symposium Special issue):820-824, 1998.

16. Stefan Schulz and Udo Hahn. Mereotopological reasoning about parts and (w)holes in bio-ontologies. In Formal Ontology in Information Systems (FOIS-2001), pages 210-221, Ogunquit, ME, 2001. ACM.

17. Stefan Schulz, Udo Hahn, and Martin Romacker. Modeling anatomical spatial relations with description logics. In JM Overhage, editor, AMIA Fall Symposium (AMIA-2000), pages 799-783, Los Angeles, CA, 2000. Hanly \& Belfus.

18. Barry Smith. The basic tools of formal ontology. In Nicola Guarino, editor, Formal Ontology in Information Systems (FOIS), pages 19-28, Amsterdam, 1998. IOS Press (Frontiers in Artificial Intelligence and Applications).

19. Barry Smith, W Ceusters, B Klagges, J Kohler, A Kumar, J Lomax, CJ Mungall, $\mathrm{F}$ Neuhause, A Rector, and C Rosse. Relations in biomedical ontologies. Genome Biology, 6(5):46, 2006.

20. C Welty and N Guarino. Supporting ontological analysis of taxonomic relationships. Data and Knowledge Engineering, 39(1):51-74, 2001.

21. ME Winston, R Chaffin, and D Hermann. A taxonomy of part-whole relations. Cognitive Science, 11:417-444, 1987. find.

22. Songmao Zhang and Olivier Bodenreider. Comparing associative concepts among equivalent concepts across ontologies. In Marius Fieschi, Enrioco Coiera, and Yu-Chuan Jack Li, editors, Medinfo-2004, pages 459-464, San Francisco, CA, 2004. IMIA, IOS Press. 